Meta

Journal des traducteurs

Translators' Journal

\title{
Le lexique anglais-français de l'industrie minière de l'Office de la langue française
}

\section{Louis-Jean Rousseau}

Volume 19, numéro 4, décembre 1974

URI : https://id.erudit.org/iderudit/004004ar

DOI : https://doi.org/10.7202/004004ar

Aller au sommaire du numéro

Éditeur(s)

Les Presses de l'Université de Montréal

ISSN

0026-0452 (imprimé)

1492-1421 (numérique)

Découvrir la revue

Citer cet article

Rousseau, L.-J. (1974). Le lexique anglais-français de l'industrie minière de l'Office de la langue française. Meta, 19(4), 189-196.

https://doi.org/10.7202/004004ar d'utilisation que vous pouvez consulter en ligne. 


\section{Le lexique anglais-français de l'industrie minière de l'Office de la langue française}

\section{INTRODUCTION}

L'Office de la langue française vient de publier le premier fascicule de son Lexique anglais-français de l'industrie minière. Nous en profitons pour exposer brièvement le sujet et expliquer nos méthodes de travail.

Le secteur des mines de l'O.L.F. a démarré à l'été 1971. Il avait mission de préparer un lexique des termes miniers. Il sagissait du premier travail d'envergure entrepris dans ce domaine au Québec.

\section{TRAVAUX DE DÉPART}

Nous nous sommes assurés, dès le début, de la collaboration d'experts de l'industrie et $\mathrm{du}$ ministère des Richesses naturelles qui nous ont apporté une assistance technique lors du dépouillement de revues spécialisées, de lexiques, de manuels et de documents d'entreprises. Nous avons pu ainsi constituer un fichier de base et une nomenclature.

Nous avons complété ce travail de dépouillement par des enquêtes orales menées dans diverses entreprises qui nous semblaient bien représenter le milieu de l'industrie minière au Québec.

À partir de ces éléments disparates, nous avons publié, après une première recherche, un lexique provisoire d'environ 8000 termes. Ce lexique provisoire n'était en fait que la liste des termes relevés lors du dépouillement et celle des équivalents français suggérés par nos premières recherches. Il n'y avait à ce stade aucune fiche terminologique pouvant appuyer notre lexique.

Cette édition provisoire a été distribuée à un nombre restreint de spécialistes : représentants de l'industrie, des universités et collèges, des syndicats ouvriers et du gouvernement. Nous avons ainsi pu obtenir leurs commentaires, leurs suggestions et leurs corrections. 
Nous avons alors entrepris le véritable travail terminologique.

\section{LE TRAVAIL TERMINOLOGIQUE}

Il s'agit de rechercher les équivalents français des termes anglais recueillis lors du dépouillement lexicographique et des enquêtes, de les vérifier et de voir si ces équivalents correspondent à l'usage afin d'établir une normalisation du vocabulaire.

Les résultats de nos recherches sont consignés sur une fiche terminologique qui comprend, pour chaque terme, la source, une définition et un contexte, et dans la langue de départ et dans la langue d'arrivée (voir fiche).

\section{A. Documentation ${ }^{1}$}

Pour la rédaction de nos fiches terminologiques, nous disposons de différentes sources documentaires que nous pouvons classer de la façon suivante : 1 . Dictionnaires généraux et dictionnaires techniques généraux; 2 . Dictionnaires et lexiques spécialisés; 3 . Manuels et périodiques spécialisés; 4. Documents d'entreprises et publicité.

1. Dictionnaires généraux et dictionnaires techniques généraux. - Avec l'expérience, on s'aperçoit rapidement que les dictionnaires généraux ne sont guère d'une grande utilité pour le travail terminologique spécialisế ; ils contiennent rarement plus de $30 \%$ du vocabulaire technique recherché.

Nous utilisons les dictionnaires techniques généraux pour les recherches concernant le vocabulaire commun à plusieurs techniques. Leur utilisation est restreinte du fait que les auteurs ne mentionnent pas toujours le domaine d'application, ce qui laisse le lecteur perplexe quant au choix d'un équivalent français.

2. Dictionnaires et lexiques spécialisés. - Il existe déjà un certain nombre de dictionnaires et de lexiques spécialisés dans le domaine de l'industrie minière et métallurgique.

Les dictionnaires unilingues du vocabulaire minier nous permettent de bien définir les notions, dans chaque langue ; leur apport est donc très important pour les fiches terminologiques.

Les lexiques spécialisés (bilingues ou multilingues) sont d'origine et de qualité diverses. Leur nomenclature n'est jamais exhaustive et le choix des équivalents français varie souvent d'un lexique à l'autre. Pour illustrer ce fait, voici quelques exemples :

Ce tableau illustre assez bien la non-concordance entre les divers lexiques. La traduction est assez uniforme pour belt conveyor : les deux formes «convoyeur à bande » et «transporteur à bande» sont utilisées à part égale dans le monde industriel, mais dans le domaine des mines, on emploie presque exclusivement l'expression « convoyeur à bande ».

1. On trouvera, à la fin de notre lexique, une bibliographie des principales sources que nous avons utilisées. 

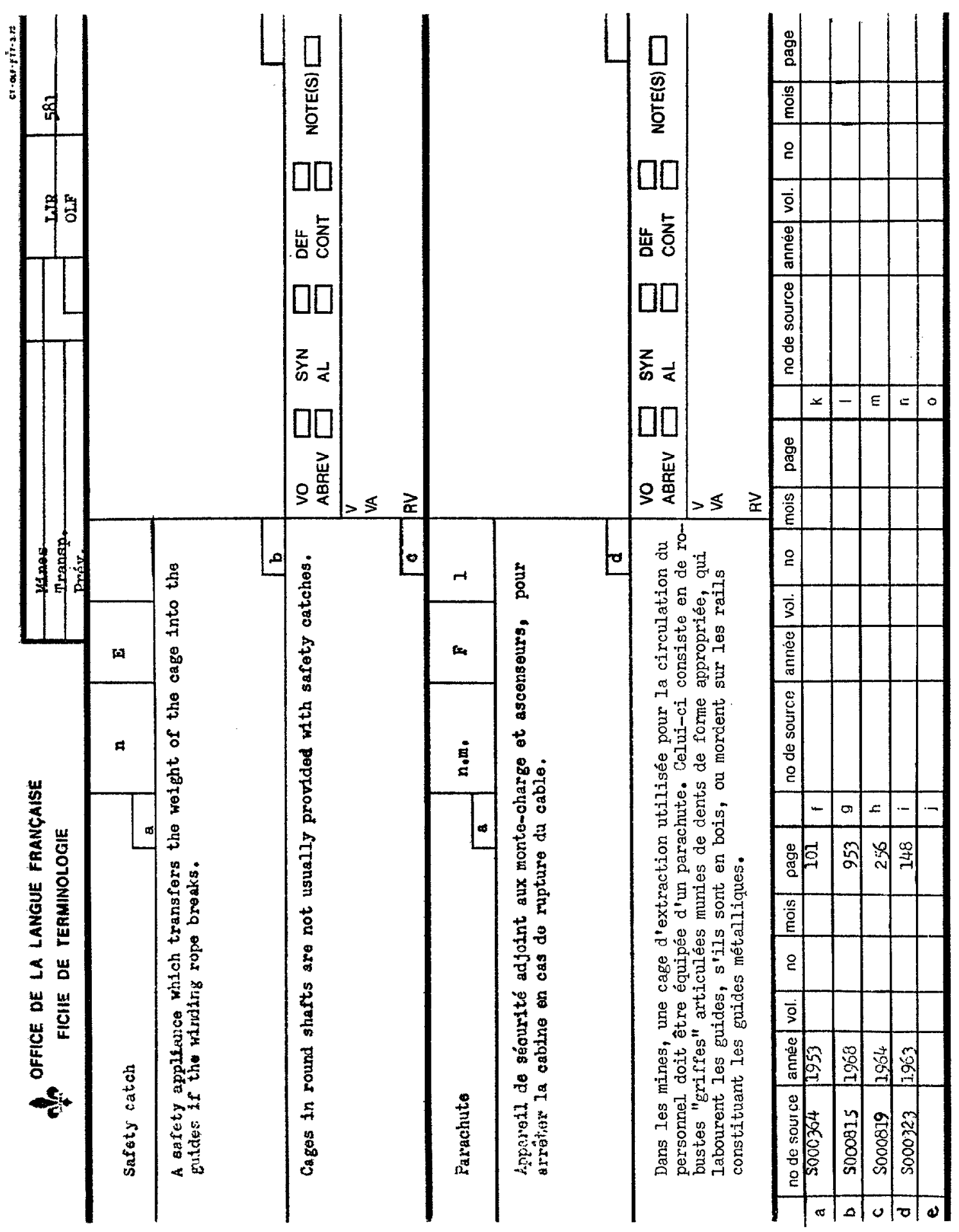


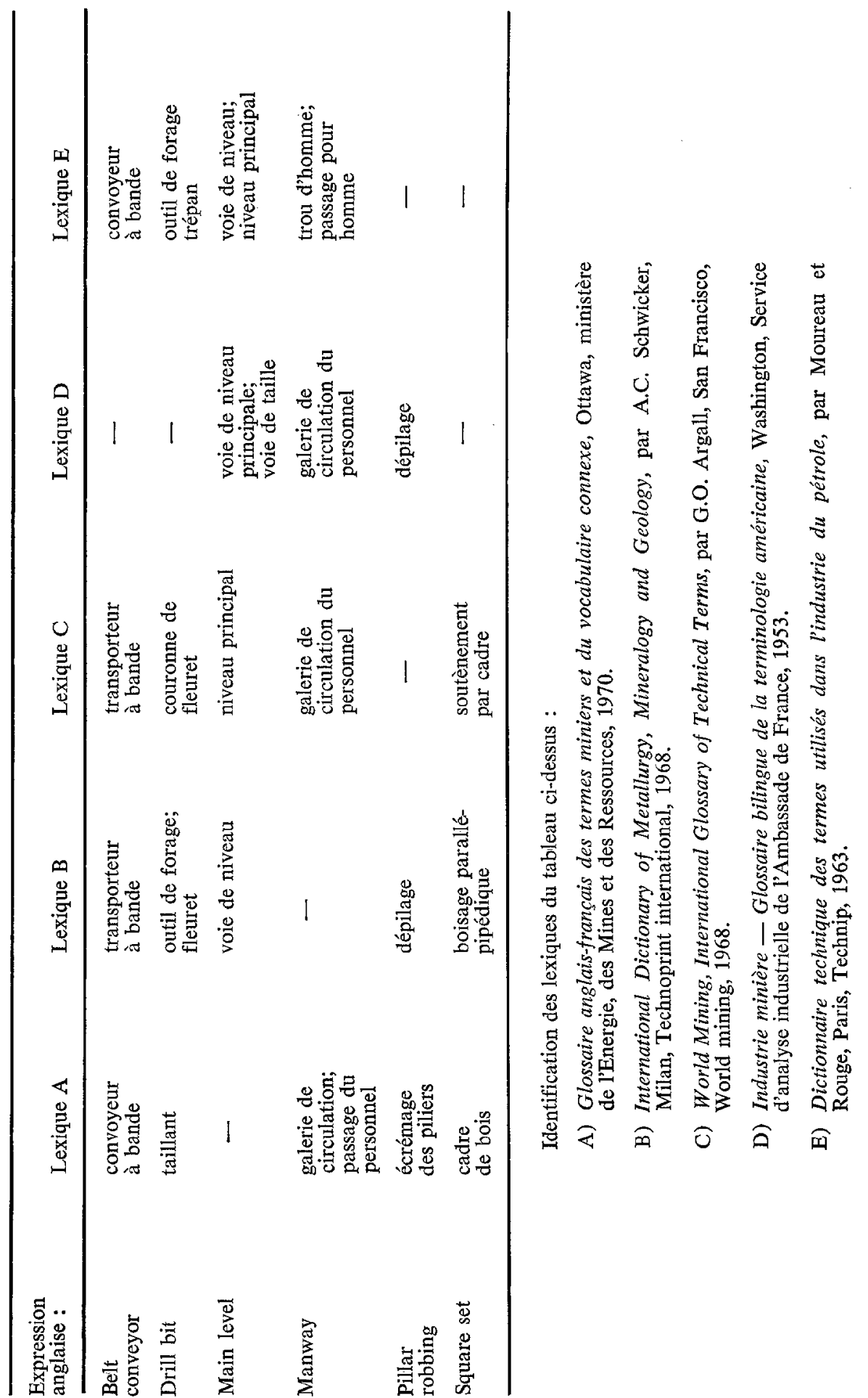


Il n'en est pas de même pour d'autres termes : aucune des traductions de square set qui figurent sur le tableau précédent n'est satisfaisante; aucune des expressions mentionnées n'est en usage, que ce soit dans la langue orale ou dans la langue écrite ; on utilise plutôt les expressions « chambre charpentée » et «cube de soutènement $\gg$.

Les quelques exemples choisis dans divers lexiques spécialisés nous montrent le peu d'uniformité du vocabulaire technique minier. Certains termes français relevés dans des lexiques ne sont pas du tout utilisés par les spécialistes dans leur travail quotidien. Nous avons parfois l'impression de nous trouver en face de traductions approximatives. Les lexiques ne sont jamais des documents de première main : les informations proviennent de sources disparates et il n'est pas rare de voir circuler les mêmes erreurs de lexique en lexique. Nous ne pouvons donc pas nous limiter à la comparaison des solutions proposées par les différents lexiques qui sont à notre disposition : il est nécessaire, croyons-nous, de trouver d'autres sources pour vérifier les équivalents français suggérés par les lexiques ou pour trouver des équivalents pour les termes que nous ne trouvons pas dans les lexiques existants.

3. Manuels et périodiques spécialisés. - Finalement, seuls les manuels et les articles de périodiques spécialisés nous permettent d'être sûrs d'un mot ou d'une expression.

Il s'agit ici de documents de première main, rédigés par des spécialistes qui utilisent quotidiennement une terminologie précise et relativement uniforme. Leur vocabulaire est confirmé par l'usage. Il est donc plus facile à normaliser.

4. Publicité et documents d'entreprises. - Il en va de même des documents publicitaires et des documents d'entreprises, quand ils proviennent de bonne source. Nous devons malheureusement utiliser avec prudence les documents qui circulent dans les entreprises au Québec ; même s'il y a des exceptions, ce sont en général des traductions plus ou moins justes, ou encore des textes dont la terminologie laisse souvent à désirer.

\section{B. Problèmes particuliers}

Les remarques qui précèdent ne font pas mention de certains problèmes que nous avons rencontrés lors de la préparation de ce lexique. En voici quelques exemples.

1. Nous devons quelquefois donner un nom français à une nouveauté technique. Il nous faut d'abord nous assurer qu'il n'y a pas déjà d'appellation française, après quoi nous essayons de trouver un terme qui est ensuite proposé à un groupe de spécialistes. Pour notre premier fascicule, nous avons laissé tomber certains termes difficiles à traduire, étant donné que nous faisions un choix de vocabulaire. Nous en avons néanmoins traité quelques-uns. Ce fut le cas, par exemple, de l'expression down-the-hole drill; les différentes sources françaises nous donnaient des périphrases, bien descriptives, mais nullement satisfaisantes sur le plan termi- 
nologique. Seul un lexique canadien ${ }^{2}$ apportait une solution intéressante : « foreuse à marteau plongeur », expression que nous avons adoptée, bien que nous n'en ayons trouvé aucune autre attestation écrite. Dans ce cas, nous lui accordons, sur la fiche terminologique, la cote de pondération «français non normalisé ».

2. On rencontre de nombreux cas de synonymie; le vocabulaire minier a été créé en partie par une certaine tradition et on est alors placé devant deux ou plusieurs termes qui se rapportent à un même référent. Cependant, il ne s'agit pas. toujours de synonymie absolue; certains termes ne sont synonymes que dans des contextes précis, ou ont des connotations différentes. Nous devons cependant inclure ces synonymes dans notre lexique dans le but d'informer le lecteur de leur existence. Pour limiter l'étendue de ces séries synonymiques, nous avons fait un choix des expressions les plus courantes, en tenant compte de l'usage québécois, et nous proposons ce choix aux utilisateurs du lexique.

Enfin, nous devons signaler que nous n'avons pas rencontré de problèmes insolubles : le vocabulaire minier est international, les méthodes d'exploitation étant les mêmes à travers le monde.

Avant de publier ce fascicule, nous avons soumis le manuscrit une dernière fois à un groupe restreint de spécialistes de l'industrie minière, et nous avons pu obtenir des commentaires et apporter quelques corrections à notre texte.

\section{Mission terminologique}

Dans le but de compléter la préparation de ce premier fascicule, nous avons effectué une mission terminologique en France. En compagnie de quelques ingénieurs de l'industrie minière québécoise et de notre consultant du ministère des Richesses naturelles, nous avons visité plusieurs exploitations minières en diverses régions de la France; cette mission nous a permis d'effectuer des enquêtes supplémentaires et d'enrichir notre documentation française. Nous y avons également trouvé la solution à divers problèmes de terminologie, en discutant avec des ingénieurs français.

\section{PRÉSENTATION DU LEXIQUE}

Étant donné l'ampleur de la nomenclature de base, nous avons décidé de publier le lexique sous forme de fascicules spécialisés. Cela nous permet de mettre à la disposition du public une première partie de nos recherches pendant que nous poursuivons le travail terminologique sur d'autres parties de la nomenclature.

Ce premier fascicule comprend environ 500 termes (les plus courants) se rapportant surtout à l'exploitation proprement dite. Le choix de ces termes a été effectué à la suite d'un sondage auprès des spécialistes que nous avons pu consulter. Nous avons préféré faire un lexique plutôt qu'un vocabulaire (avec définitions), puisque notre clientèle est constituée de spécialistes (ingénieurs, techniciens et mineurs) qui connaissent bien la matière et pour qui la définition des termes ne pose pas de problème. Le lexique se présente donc ainsi :

2. A.S. Romaniuk, Glossaire anglais-français des termes miniers et du vocabu'aire connexe. Ottawa, ministère de l'Energie, des Mines et des Ressources, 1970. 


\begin{tabular}{lllr}
\hline ANGLAIS & \multicolumn{1}{c}{ FRANÇAS } & $\begin{array}{r}\text { DOMAINES } \\
\text { D'EMPLOI }\end{array}$ \\
\hline 44 & BACKFILL & REMBLAI & 11 \\
45 & BACKFILL MATERIAL & $\begin{array}{l}\text { MATÉRIAU DE REMBLAYAGE } \\
\text { REMBLAI }\end{array}$ & 11 \\
46 & BACKFILLING & REMBLAYAGE & 11 \\
47 & BACKFILLING, HYDRAULIC & REMBLAYAGE HYDRAULIQUE & 11 \\
48 & BACKFILLING SHIFT & POSTE DE REMBLAYAGE & 11,19 \\
49 & BACKFILLING WITH & REMBLAYAGE PAR RACLAGE & 11 \\
& SCRAPER & REMBLAYAGE PAR SCRAPER & \\
50 & BACKHOLE & TROU DE COURONNE & 4,5 \\
& & TROU DE TOIT & \\
& & TROU DE VOUTE & \\
\hline
\end{tabular}

Nous avons spécifié le domaine d'emploi pour éviter certaines ambiguïtés dues à des cas de polysémie. À cette fin, nous avons classé le vocabulaire en regroupant les domaines en 20 sections qui décrivent le cheminement approximatif d'une exploitation minière. Voici la liste de ces domaines :

1. Géologie - Minéralogie - Pétrographie — Gîtologie.

2. Recherche des gisements.

3. Topographie - Dessin.

4. Forage - Sondage.

5. Explosifs.

6. Travaux préparatoires - Chantiers.

7. Voies de pénétration et de circulation.

8. Dépilage et abattage du minerai.

9. Chargement des produits - Déblayage.

10. Transport des produits, des matériaux et du personnel — Extraction.

11. Pressions de terrain - Mécanique des sols - Soutènement.

12. Aérage.

13. Exhaure.

14. Minéralurgie - Métallurgie.

15. Énergie (génération, transport, utilisation).

16. Outillage ou matériel d'usages variés.

17. Engins d'usages variés.

18. Prévention - Sécurité.

19. Organisation du travail - Appellations d'emplois.

20. Divers - Vocabulaire minier général.

Nous avons adjoint au lexique un index des termes français, ce qui permettra d'utiliser le lexique dans les deux sens. 
Dans le lexique comme dans l'index, nous avons mis en vedette tous les mots significatifs, ce qui permet d'une part de retrouver les expressions plus facilement, et d'autre part, de grouper les mots de même famille; pour faciliter ce regroupement, nous avons adopté la classification alphabétique discontinue.

\section{CONCLUSION}

La préparation de ce premier fascicule de notre lexique nous a permis de roder nos méthodes et de trouver des moyens d'accélérer le travail, tout en conservant les mêmes critères de qualité.

Ainsi, nous expérimentons actuellement, pour la préparation de notre deuxième fascicule, une méthode de dépouillement systématique de la documentation (mots et contextes). Nous avons choisi cette méthode à cause de la lenteur du procédé utilisé pour la rédaction des fiches terminologiques du premier fascicule : nous procédions mot par mot, à partir de la nomenclature préétablie, cc qui nous amenait à repasser plusieurs fois les mêmes ouvrages à la recherche d'attestations en langue anglaise et en langue française.

Nous attendons avec impatience les réactions du public, car nous espérons recevoir des commentaires qui nous permettront d'améliorer la qualité de nos travaux. Signalons pour terminer que la première édition de notre lexique a été tirée à 20000 exemplaires et qu'il est distribué gratuitement aux personnes qui en font la demande. 